\title{
Restoring the Esthetics for a Cleft Palate Patient by Using Modified Preparation and Pink Porcelain
}

\author{
Ebrahim Fihaid Alsubaiy ${ }^{1,2}$, Hisham Abdullah Mously ${ }^{3}$
}

\begin{abstract}
Aim: This report describes the challenges experienced in a unique case involving dental esthetics, and the treatment provided to achieve satisfactory conservative treatment.

Background: Even minor aspects of dental esthetics can significantly affect a patient's quality of life. Moreover, the patient's level of satisfaction with rehabilitative dentition and prosthetics affects oral health, daily living, and dental perceptions. It is not unusual for clinicians to encounter cases with complex esthetics that have affected the patient's life. Such cases need careful treatment planning to meet the patient's expectations. Conservative treatment should be the clinician's goal, because complex treatment may result in negative outcomes.

Case description: Here, the patient exhibited severe bone loss due to cleft palate, as well as improper positioning of the teeth. He could not be treated with implants because of poor bone quality and quantity. Orthodontic treatment was performed, but did not improve the outcome. A wax-up was made for each of the three possible options, and then transferred to the patient's mouth by making a mock-up. This allowed the patient to clearly understand the results of each option. After selecting an option, we performed the procedure on the cast before it was undertaken in the patient's mouth. This helped us to precisely determine the preparation needed for all teeth. Teeth were saved where possible to preserve the remaining bone. The lost bone was replaced with pink porcelain. Treatment was conservative and the results were optimal for the patient and our clinical team.
\end{abstract}

Conclusion: In some cases, clinicians may have few options available to achieve satisfactory results. However, the use of appropriate diagnostic tools, data collection, and conservative treatment procedures can help achieve these goals.

Clinical significance: Fabrication of wax-ups and mock-ups can help the clinician and patient predict the results of dental treatment and ensure a conservative procedure.

Keywords: Esthetics, Maxillary advancement, Prosthetics, Wax-up.

The Journal of Contemporary Dental Practice (2019): 10.5005/jp-journals-10024-2572

\section{INTRODUCTION}

Even minor aspects of dental esthetics can have a direct and significant effect on a patient's quality of life. 'Additionally, the patient's level of satisfaction with rehabilitative dentition and prosthetics can positively affect oral health while improving the aspects of daily living and dental perceptions. ${ }^{2}$ Dentists must form an adequate treatment plan and maintain the appropriate skills needed to provide satisfactory and conservative treatment. In this report, I have described my experiences with the aspects of dental esthetics in a unique and challenging dental case.

\section{Case Description}

A 22-year-old man was referred from the surgery clinic to the prosthodontics dental clinic at our university after clinicians determined that orthodontic treatment would not be feasible. The patient had a cleft lip and palate for which he had undergone many surgical treatments coordinated between the orthodontics and surgery departments, beginning at 4 years of age. However, surgical treatments, including bone grafting or implants, were not possible.

This patient presented to our clinic with many esthetic problems, which were complicated by the contraindication for orthodontic and surgical treatments. The problems included missing central and lateral incisors; retention of a deciduous tooth (upper right lateral incisor); decayed central incisor in the dental midline; incorrect arrangement of the decayed central incisor, upper left canine, and upper left first premolar; asymmetry of the gingival levels of the anterior teeth; failure of the maxillary teeth to follow
${ }^{1}$ Department of Prosthodontics, King Khalid University, Abha, Kingdom of Saudi Arabia

${ }^{2}$ Prosthodontics Saudi Board Program, College of Dentistry, King Abdulaziz University, Jeddah, Kingdom of Saudi Arabia

${ }^{3}$ Department of Prosthodontics, King Abdulaziz University, Jeddah, Kingdom of Saudi Arabia

Corresponding Author: Ebrahim Fihaid Alsubaiy, Department of Prosthodontics, King Khalid University, Abha, Kingdom of Saudi Arabia, Phone: +96 6565783333, e-mail: Ebrahim-fs@hotmail.com

How to cite this article: Alsubaiy EF, Mously HA. Restoring the Esthetics for a Cleft Palate Patient by Using Modified Preparation and PinkPorcelain. J Contemp Dent Pract 2019;20(5):645-649.

Source of support: Nil

Conflict of interest: None

Patient consent: Obtained

the curvature of the lower lip; and failure of the dental midline to coincide with the facial midline (Fig. 1).

It was determined that the decayed central incisor could not be restored, and the deciduous tooth was mobile, with a short root. Accordingly, these teeth would require extraction. After fabrication of diagnostic casts, we made two wax-ups for the purpose of treatment planning. The first wax-up was made after removing the deciduous tooth and decayed central incisor from the cast. Here, the upper left canine was reshaped as a central incisor, and the upper left first premolar was reshaped as a lateral incisor with the intent to restore the teeth with fixed partial dentures (FPDs) and abutment teeth (i.e., the upper right 

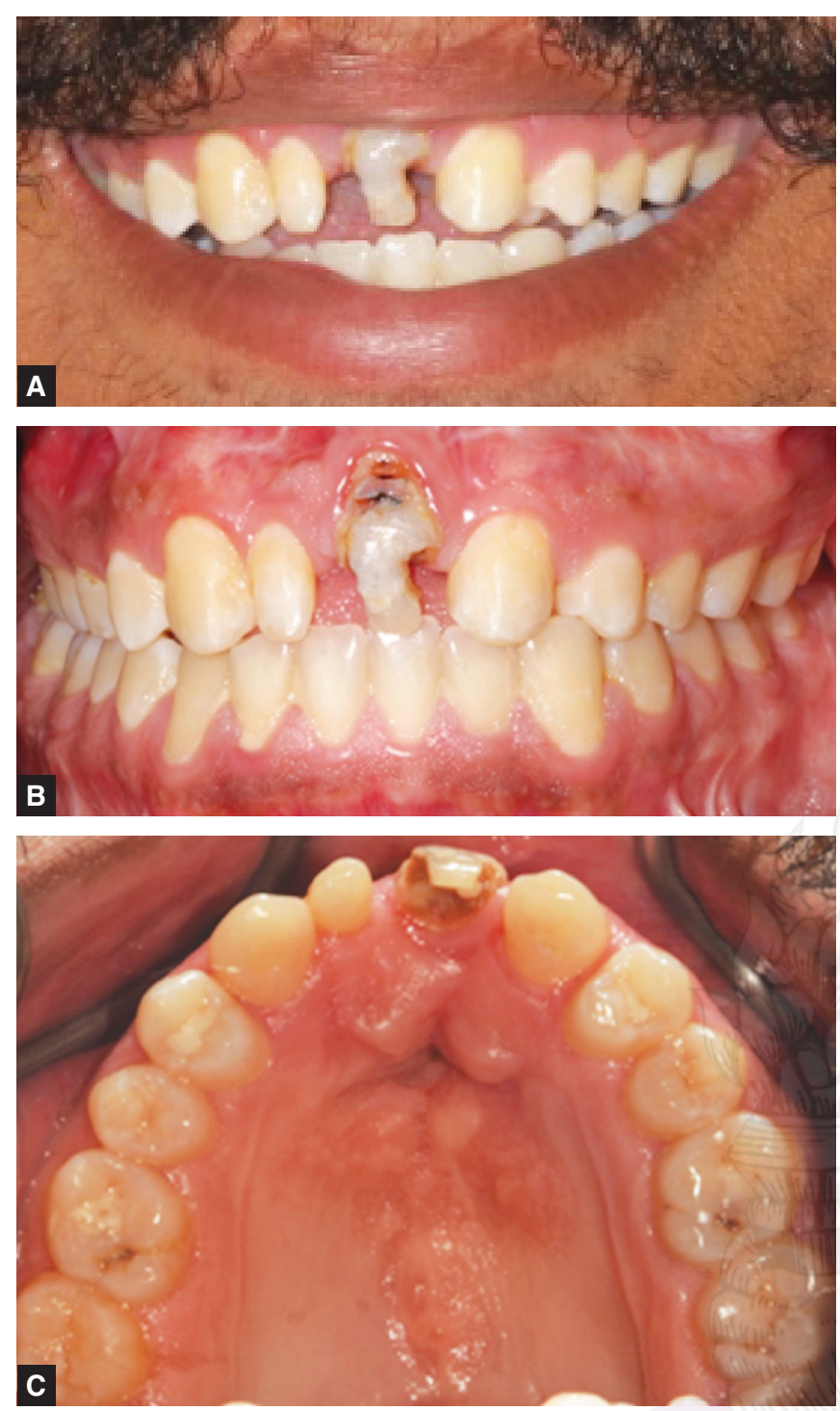

Figs 1 A to C: (A) Preoperative smile view; (B) Preoperative frontal view; (C) Preoperative occlusal view

canine, upper left canine, and upper left first premolar). The second wax-up was made after removing the deciduous tooth, decayed central incisor, and upper left canine from the cast; the latter was removed to provide a well-distributed space in which the teeth could be arranged properly. Subsequently, the upper left premolar was reshaped as a canine. This plan involved restoring the teeth with an FPD and abutment teeth (i.e., upper right canine and upper left first premolar). ${ }^{3,4}$

Subsequently, two mock-ups were made corresponding to the two wax-ups (Fig. 2). The use of mock-ups is an effective method for estimating procedural outcomes and can help the clinician and patient determine the most appropriate treatment option. In this case, the patient was not satisfied with the first option because the dental midline did not coincide with the facial midline. However, the patient was highly satisfied with the second option, which required the extraction of a sound tooth (upper left canine).

Next, a trial was performed on the cast in an attempt to achieve a simultaneously conservative and satisfactory treatment. First, a diagnostic cast was fabricated and a putty index made according to the wax-up with the ideal arrangement of teeth. Using the putty index as a preparation guide, the teeth were prepared to evaluate the
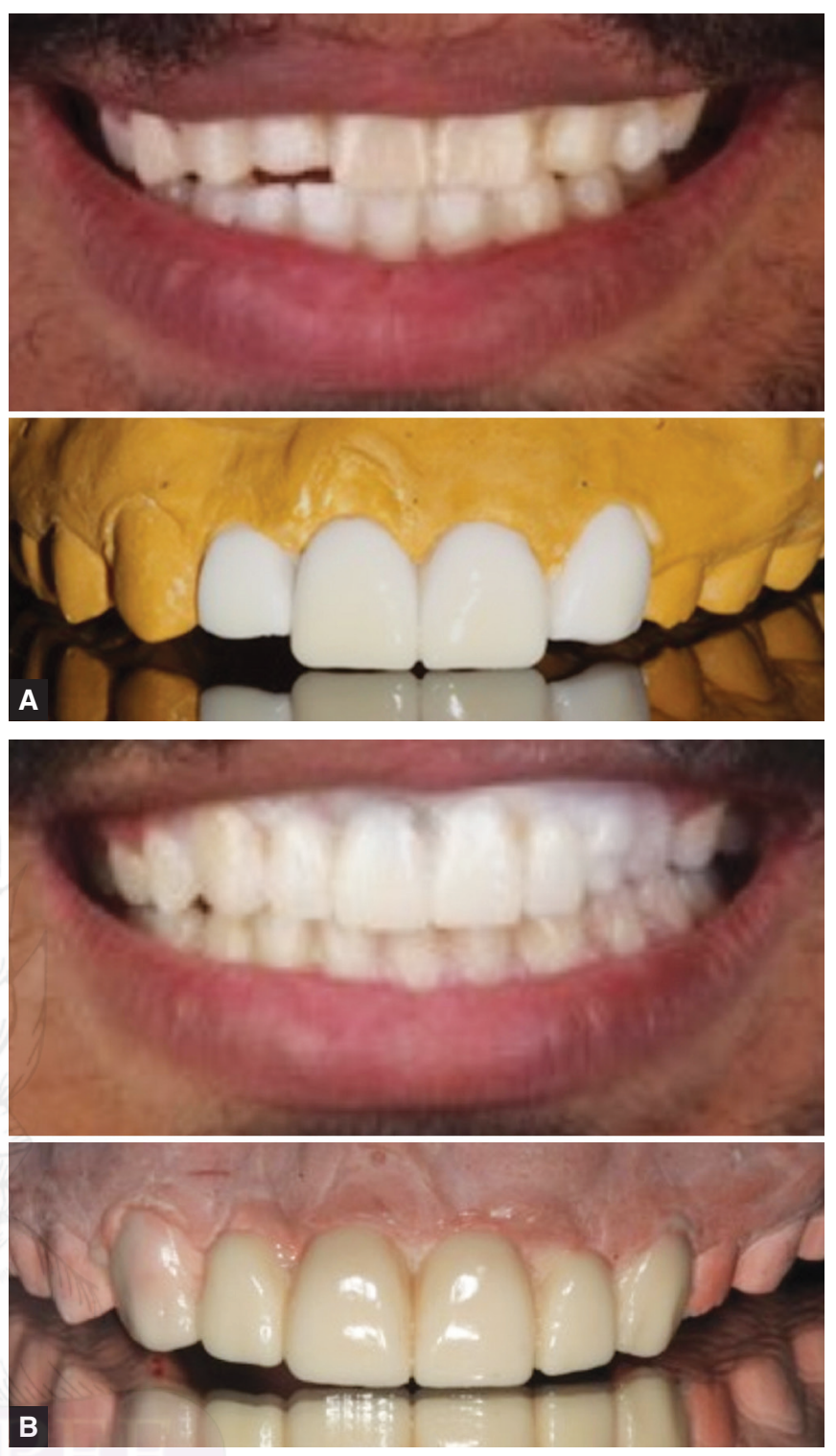

Figs $2 A$ and B: (A) Mock-up and wax-up for the first option; (B) Mock-up and wax-up for the second option

possibility of saving the upper left canine. This evaluation revealed that the canine could be saved via significant preparation of its distal surface and the mesial surface of the premolar, which created a space for the upper left lateral incisor (Fig. 3). The abutment teeth (upper right canine, upper left canine, and upper left first premolar) were found to be non-vital and were subjected to root canal treatment.

The teeth were prepared using the guides (Fig. 4), and a temporary FPD was constructed before extracting the deciduous tooth (upper right lateral incisor) and the central incisor (i.e., decayed tooth) to reduce psychological shock. An evaluation of the patient's smile revealed the following: the upper left first and second premolars required crown lengthening to resemble a canine and achieve a harmonious gingival level, respectively; ceramic veneers were required to mask the discoloration of the upper left second premolar and upper right first premolar and the misalignment of the latter; and bleaching of the mandibular teeth was needed. ${ }^{5}$

The indicated crown lengthening was performed for the upper left first and second premolars, and the final preparation of 

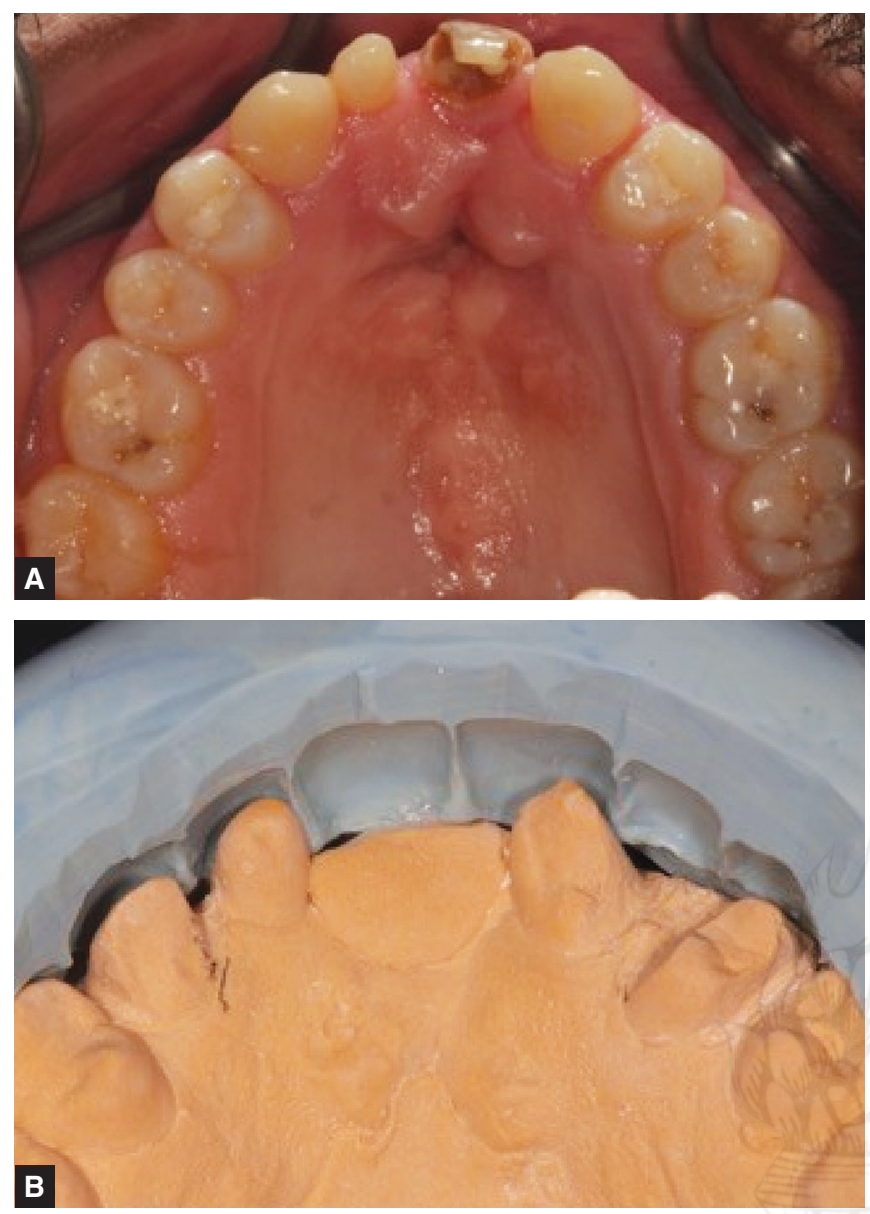

Figs 3A and B: (A) Occlusal view for the maxillary arch; (B) Preparation for the teeth by using the putty index for the second wax-up

the abutment teeth was performed. A final impression was made using polyvinyl siloxane (PVS) impression material, and the FPD was constructed using multilayer zirconia (Amann Girrbach). ${ }^{6-12}$ After the patient tested the FPD, the upper right first premolar and the upper left second premolar were subjected to preparation, and the final impression was made with the FPD left in place to ensure that the accurate proximal contacts would be achieved.

After delivery of the final prosthesis and bleaching of the mandibular teeth (Fig. 5), the gingival levels were corrected via crown lengthening and by replacing the soft tissue with pink porcelain (Fig. 5). In this manner, the goal of coinciding the dental midline with the facial midline was achieved. Well-arranged maxillary teeth that followed the curvature of the lower lip were also achieved, which is one of the requirements for a beautiful smile (Fig. 6). After treatment, the patient had properly sized teeth with distributions that followed the curvature of the lower teeth.

Postoperatively, the patient was provided with instructions regarding maintenance of the restorations and the health of the surrounding tissues.

Regular follow-up visits were scheduled to evaluate the patient's oral hygiene and the status of the restorations.

\section{Discussion}

Dental esthetics can have a significant effect on a patient's quality of life. However, some cases of dental treatment, such as the present
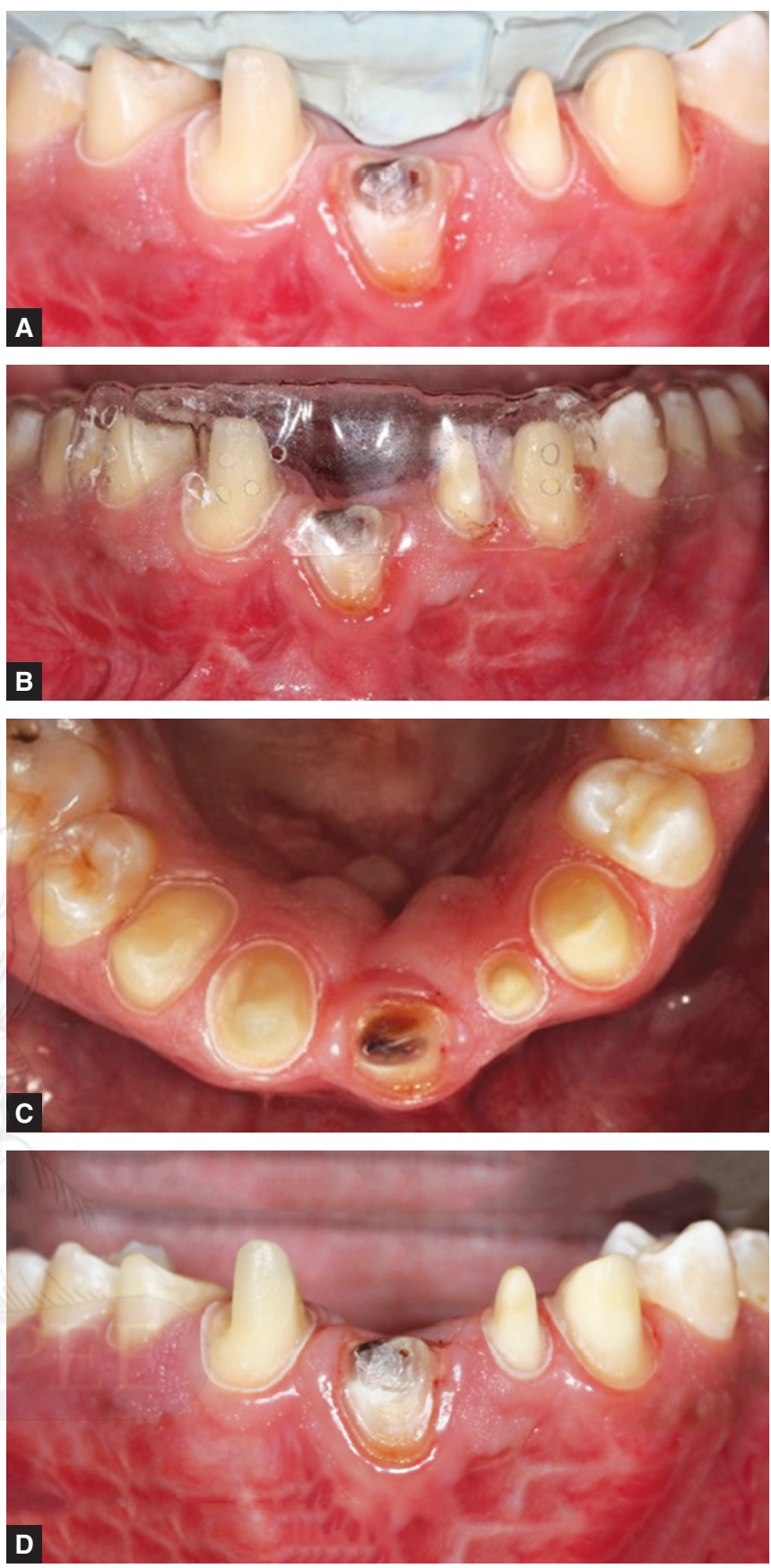

Figs 4A to D: (A) Preparation of the teeth by using putty index; (B) Preparation of the teeth by using vacuum shell; (C) Occlusal view for the prepared teeth; (D) Frontal view for the prepared teeth

case, present unique challenges and clinicians may be faced with few options for achieving a satisfactory result. One of the features of the present case was the poor quantity and quality of bone. The patient had undergone several dental surgical procedures, which resulted in the deterioration of the bone quality. This limited the available treatment options and precluded the possibility of using dental implants, because dental implants can be used to replace missing teeth only when the quality and quantity of bone are optimal. Another option to treat such cases is a combination of surgical and orthodontic treatments to redistribute the teeth in proper positions. In a similar report, Boyne and Sands described 

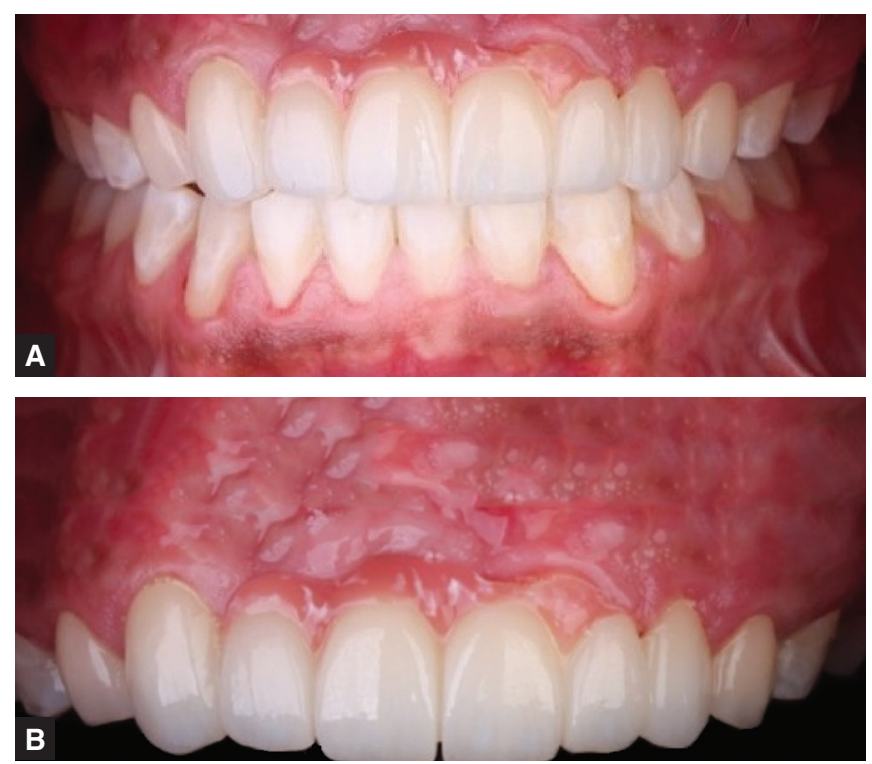

Figs 5A and B: (A) After cementation of final prosthesis and bleaching of lower teeth; (B) After correction of the gingival levels

residual bony anterior palatal and alveolar deformities of cleft palate patients. They studied the effects of grafting on osseous repair, using marrow and cancellous bone grafting, and found that the amount of osseous tissue diminished with increasing age of the patient. Therefore, in adult patients, such defects were periodontal problems that were impacted by increasing the periodontal pocket depth. ${ }^{13}$ In the present case, because maxillary advancement and extended orthodontic treatments had been performed since the patient was 4 years of age, there was no possibility of further benefit from orthodontic treatment. In another study, Marcusson et al. evaluated the satisfaction of cleft palate-treated patients regarding their facial appearance. They concluded that these treated patients were unsatisfied with their facial appearance, and desired additional operations. ${ }^{14}$ Dental esthetic was an issue of importance for subjects who were unsatisfied. Notably, improvement of dental esthetic through the use of a suitable prosthesis can improve patients' quality of life. Another option for the treatment of such patients is the application of a removable partial denture. Meşe and Özdemir performed treatment for a cleft palate patient, which involved the use of a removable partial denture. ${ }^{15}$ The disadvantage of this approach is that patients may not be satisfied with a removable partial denture, due to the inherent appearance of clasps and loss of retention. In the present case, the elimination of treatment options, such as bone grafting, implant placement, and orthodontic treatment, further complicated the situation, because bone and soft tissue loss would affect the esthetics. Treatment of this patient by modifying the preparation and using pink porcelain enabled replacement of the missing soft tissue and redistribution of the teeth in proper positions. To achieve the optimal result in such cases, accurate diagnosis and collection of relevant information is imperative. Proper data analysis helps the clinician to achieve treatment goals and helps the patient to understand the predicted final results. In the present case, the fabrication of a wax-up for each option, as well as representation of different surgical results through the use of mock-ups, helped us to achieve the optimal treatment outcome. This treatment was conservative when compared with other complicated and extended procedures. Moreover, in such
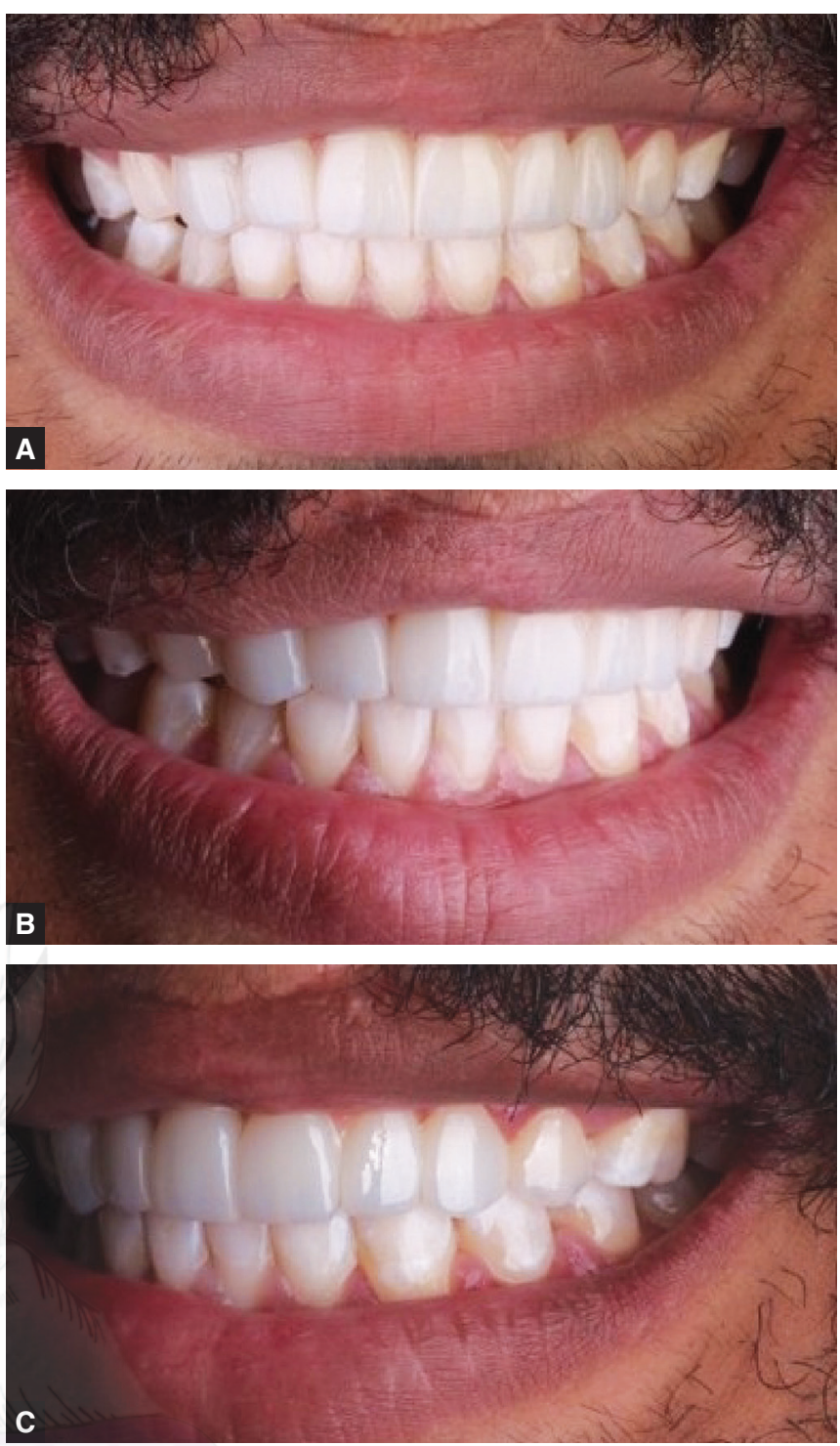

Figs 6A to C: (A) Frontal smile; (B) Smile (right side); (C) Smile (left side)

cases, conservative procedures have a higher probability of success, compared to other procedures (e.g., surgical or orthodontic).

\section{Limitations of this TECHNIQUE}

To apply this technique, sufficient abutments are needed, which enable the FPD to be used. In most cleft palate patients, many teeth are missing, such that the FPD requires a long span of multiple teeth. Weak abutments that are periodontally compromised or exhibit a poor crown-to-root ratio will prevent the use of this technique. Additionally, good oral hygiene is an important consideration, because the most frequent complication for patients with FPDs is recurrent caries.

\section{Conclusion}

In some cases of dental treatment, it is challenging for clinicians to achieve satisfactory results when few treatment options are available. However, adequate data collection, conservative treatment procedures, and use of appropriate diagnostic tools can help clinicians to achieve treatment goals. 


\section{Clinical Significance}

Some techniques help clinicians to design an effective treatment plan, whereas others aid in the prediction of the final outcome. Before treatment, the clinician should perform the preparation needed to achieve optimal treatment outcome.

\section{ACKnowledgments}

This study did not receive any external funding.

\section{References}

1. Klages U, Bruckner A, et al. Dental aesthetics, self-awareness, and oral health-related quality of life in young adults. Eur J Orthod 2004 Oct;26(5):507-514.

2. Isiekwe GI, Sofola OO, et al. Dental esthetics and oral health-related quality of life in young adults. Am J Orthod Dentofacial Orthop 2016 Oct;150(4):627-636. DOI: 10.1016/j.ajodo.2016.03.025.

3. Sadowsky SJ. An overview of treatment considerations for esthetic restorations: a review of the literature. J Prosthet Dent 2006 Dec;96(6):433-442.

4. Tan $\mathrm{K}$, Pjetursson BE, et al. A systematic review of the survival and complication rates of fixed partial dentures (FPDs) after an observation period of at least 5 years. Clin Oral Implants Res 2004 Dec;15(6): 654-666. DOI: 10.1111/j.1600-0501.2004.01119.x.

5. Turgut $\mathrm{S}$, Bagis $\mathrm{B}$, et al. Achieving the desired colour in discoloured teeth, using leucite-based cad-cam laminate systems. J Dent 2014 Jan;42(1):68-74. DOI: 10.1016/j.jdent.2013.10.018.

6. Pjetursson BE, Sailer I, et al. All-ceramic or metal-ceramic toothsupported fixed dental prostheses (FDPs)? A systematic review of the survival and complication rates. Part II: multiple-unit FDPs. Dent Mater 2015 Jun;31(6):624-639. DOI: 10.1016/j.dental.2015.02.013.

7. Raigrodski AJ, Hillstead MB, et al. Survival and compli-cations of zirconia-based fixed dental prostheses: a systematic review. J Prosthet Dent 2012 Mar;107(3):170-177. DOI: 10.1016/S00223913(12)60051-1.

8. Olsson KG, Fürst $B$, et al. A long-term retrospective and clinical follow-up study of In-Ceram Alumina FPDs. Int J Prosthodont 2003 Mar-Apr;16(2):150-156.

9. Raigrodski AJ, Chiche GJ, et al. All-ceramic fixed partial dentures. Part III: Clinical studies. J Esthet Restor Dent 2002;14(5):313-319.

10. Christel $P$, Meunier $A$, et al. Mechanical properties and short-term in vivo evaluation of yttrium-oxide-partially-stabilized zirconia. J Biomed Mater Res 1989 Jan;23(1):45-61. DOI: 10.1002/jbm.820230105.

11. Guazzato M, Albakry M, et al. Strength, fracture toughness and microstructure of a selection of all-ceramic materials. Part Il. Zirconiabased dental ceramics. Dent Mater 2004 Jun;20(5):449-456. DOI: 10.1016/j.dental.2003.05.002.

12. Sailer I, Gottnerb J, et al. Randomized controlled clinical trial of zirconiaceramic and metal-ceramic posterior fixed dental prostheses: a 3-year follow-up. Int J Prosthodont 2009 Nov-Dec;22(6):553-560.

13. Boyne PJ, Sands NR. Combined orthodontic-surgical management of residual palato-alveolar cleft defects. Am J Orthod 1976 Jul;70(1): 20-37.

14. Marcusson A, Paulin G, et al. Facial appearance in adults who had cleft lip and palate treated in childhood. Scand J Plast Reconst Surg Hand Surg 2002 Jan;36(1):16-23.

15. Meşe A, Özdemir E. Removable partial denture in a cleft lip and palate patient: a case report. J Korean Med Sci 2008 Oct;23(5):924-927. DOI: 10.3346/jkms.2008.23.5.924. 